\title{
Consistency based completion approaches of incomplete preference relations in uncertain decision contexts
}

\author{
Raquel Ureña, Francisco Chiclana and Enrique Herrera-Viedma
}

\begin{abstract}
Uncertainty, hesitation and vagueness are inherent to human beings when articulating opinions and preferences. Therefore in decision making situations it might well be the case that experts are unable to express their opinions in an accurate way. Under these circumstances, various families of preference relations (PRs) have been proposed (linguistic, intuitionistic and interval fuzzy PRs) to allow the experts to manifest some degree of hesitation when enunciating their opinions. An extreme case of uncertainty happens when an expert is unable to differentiate the degree up to which one preference is preferred to another. Henceforth, incomplete preference relations are possible. It is worth to bear in mind that incomplete information does not mean low quality information, on the contrary, in many occasions experts might prefer no to provide information in other to keep consistency. Consequently mechanism to deal with incomplete information in decision making are necessary. This contribution presents the main consistency based completion approaches to estimate incomplete preference values in linguistic, intuitionistic and interval fuzzy PRs.
\end{abstract}

\section{INTRODUCTION}

A comparative study between different alternative preference elicitation methods reported in [1] concluded that pairwise comparison methods are more accurate than non-pairwise methods because the formers ease the experts to present their preferences focusing exclusively on two alternatives at a time. Nevertheless, their associated drawback, is that some experts might not been able to discriminate the degree up to which some of the options are better than others, and as a consequence incomplete preferences are provided.

In these situations, some approaches propose to discard or rate more negatively the experts that provide incomplete preferences [2] under the premise that a good solution to a decision making problem cannot be achieved from incomplete information. On the contrary, empirical evidence suggests that the incomplete PR derived from the random deletion of as much as $50 \%$ of the elements of a complete pairwise PR

Raquel Ureña is with the Department of Computer Science and Artificial Intelligence, University of Granada, Granada, Spain (email: raquel@decsai.ugr.es).

Francisco Chiclana is with the Centre for Computational Intelligence $(C C I)$ and the DMU Interdisciplinary Group in Intelligent Transport Systems (DIGITS), Faculty of Technology, De Montfort University, Leicester, UK (email: chiclana@dmu.ac.uk).

Enrique Herrera-Viedma is with the Department of Computer Science and Artificial Intelligence, University of Granada, Granada, Spain (email: viedma@decsai.ugr.es).

This research work has been developed with the financing of FEDER funds in FUZZYLING-II Project TIN2010-17876,TIN2013-40658-P, the Andalusian Excellence Projects TIC-05299 and TIC-5991, the mobility grant program awarded by the University of Granada 's International Office and the University of Granada 'Strengthening through Short-Visits' (Ref. GENIL-SSV 2015) programme. provides good results without compromising accuracy [3]. Indeed, incomplete information does not mean low quality information and therefore these approaches could delete useful information leading to biased and inaccurate results. Hence approaches to complete or estimate the missing information becomes necessary.

Equally important is the fact that subjectivity, imprecision and vagueness in the articulation of opinions pervade real world decision applications, and it becomes hard for the experts to articulate their preferences by means of exact values. To overcome this issue two main mathematical frameworks have been proposed:

- Numeric PRs that allow to express preferences with a greater degree of uncertainty, namely the Interval valued PRs (IVPRs) and the Intuitionistic PRs (IPRs). Both have been proved to be isomorphic in [4].

- Linguistic PRs (LPRs) allow experts to express their opinions by means of a finite set of linguistic labels. The main advantage of this approach is that enables the users to express their opinions by means of words. Nevertheless this approach could lead to loss of information because of the linguistic approximations. Furthermore choosing the cardinality of the linguistic term set is another issue to bear in mind since words could mean different things to different people [5].

In this contribution we present a critical study of the main consistency based completion approaches to estimate incomplete preference values when dealing with IPRs, IVPRs and LPRs. The rest of this contribution is organized as follows: Section II presents the preliminary concepts that will be needed in subsequent sections. This include the presentation of the main preference representation formats dealt with as well as the concept of consistency of preferences. Section III presents consistency based completion approaches to estimate incomplete preference values for the preference relations previously presented. Finally, conclusions are drawn and future research work on this area are presented in Section IV.

\section{PREFEREnCE RELATIONS AND CONSISTENCY OF PREFERENCES}

When carrying out decision-making processes experts usually are required to compare a finite set of alternatives $X=\left\{x_{1}, x_{2}, \ldots, x_{n}\right\}$ with respect to a single criterion, and construct PRs. Formally, a preference relation is defined as follows:

Definition 1 (Preference Relation $(P R))$ : A preference relation $R$ is a binary relation defined on the set $X$ that is 
characterised by a function $\mu_{p}: X \times X \rightarrow D$, where $\mathrm{D}$ is the domain of representation of preference degrees provided by the decision maker.

When cardinality of $X$ is small, $R$ may be conveniently represented by an $n \times n$ matrix $R=\left(r_{i j}\right)$, with $r_{i j}=\mu_{p}\left(x_{i}, x_{j}\right)$ being interpreted as the degree or intensity of preference of alternative $x_{i}$ over $x_{j}$. There are two basic types of numeric PRs: crisp PRs [6] and [0,1]-valued PRs commonly known as Fuzzy PRs, FPRs [7]. The second type of relation is an extension of the first one, that is FPRs have crisp relations as a particular case. The elements of $R$ can also be of linguistic nature, i.e. can be any of a possible set of linguistic labels. In the following we will focus on three types of PRs: IntervalValued PRs (II-A), Intuitionistic PRs (II-B) and Linguistic PRS (II-C).

\section{A. Interval-Valued PRs}

The introduction of the concept of fuzzy set as an extension of the classical concept of set when applied to a binary relation leads to the concept of a fuzzy or $[0,1]$-valued preference relation, $P=\left(p_{i j}\right)$ [7], referred to as reciprocal preference relation (RPR) in this contribution:

Definition 2 (Reciprocal Preference Relation (RPR)): A RPR $P$ on a finite set of alternatives $X$ is characterised by a membership function

$$
\mu_{P}: X \times X \longrightarrow[0,1], \mu_{P}\left(x_{i}, x_{j}\right)=p_{i j},
$$

verifying

$$
p_{i j}+p_{j i}=1 \forall i, j \in\{1, \ldots, n\} .
$$

The following interpretation is assumed:

- $p_{i j}>0.5$ indicates that the expert prefers the alternative $x_{i}$ to the alternative $x_{j}$, with $p_{i j}=1$ being the maximum degree of preference for $x_{i}$ over $x_{j}$;

- $p_{i j}=0.5$ represents indifference between $x_{i}$ and $x_{j}$.

As Mendel stated in [8], the membership functions of fuzzy sets are subject to uncertainty arising from various sources. To reflect this uncertainty Klir and Folger proposed to blur a fuzzy set to create an interval-valued fuzzy set [9]:

Definition 3 (Interval-Valued Fuzzy Set (IVFS)): Let $\operatorname{INT}([0,1])$ be the set of all closed subintervals of $[0,1]$ and $X$ be a universe of discourse. An interval-valued fuzzy set (IVFS) $\widetilde{A}$ on $X$ is characterised by a membership function $\mu_{\widetilde{A}}: X \rightarrow \operatorname{INT}([0,1])$. An IVFS $\widetilde{A}$ on $X$ can be expressed as follows:

$$
A=\left\{\left(x, \mu_{\widetilde{A}}(x)\right) ; \mu_{\widetilde{A}}(x) \in I N T([0,1]) \forall x \in X\right\} .
$$

The application of the concept of IVFS to an RPR leads to the concept of interval-valued RPR (IVRPR), i.e. a preference relation with domain of representation of preference degrees is the set of all closed subintervals of $[0,1], D=I N T([0,1])$.

Definition 4 (Interval-Valued RPR (IVRPR)): An IVRPR $B$ on a finite set of alternatives $X=\left\{x_{1}, \ldots, x_{n}\right\}$ is characterised by a membership function $\mu_{B}: X \times X \longrightarrow \tilde{P}([0,1])$ with $\mu_{B}\left(x_{i}, x_{j}\right)=\left[b_{i j}^{-}, b_{i j}^{+}\right]$verifying $b_{i j}^{-}+b_{j i}^{+}=b_{i j}^{+}+b_{j i}^{-}=$ $1, b_{i i}^{+}=b_{i i}^{-}=0.5 \quad \forall i, j=1,2, \ldots n$.

\section{B. Intuitionistic Preference Relations}

The concept of an intuitionistic fuzzy set (IFS) was introduced by Atanasov in [10]:

Definition 5 (Intuitionistic Fuzzy Set (IFS)): An intuitionistic fuzzy set (IFS) $A$ over a universe of discourse $X$ is represented as $A=\left\{\left(x,\left\langle\mu_{A}(x), \nu_{A}(x)\right\rangle\right) \mid x \in X\right\}$ where $\mu_{A}: X \rightarrow[0,1]$, $\nu_{A}: X \rightarrow[0,1]$ and $0 \leq \mu_{A}(x)+\nu_{A}(x) \leq 1 \quad \forall x \in X$. For each $x \in X$, the numbers $\mu_{A}(x)$ and $\nu_{A}(x)$ are known as the degree of membership and degree of non-membership of $x$ to $A$, respectively.

An IFS becomes a FS when $\mu_{A}(x)=1-\nu_{A}(x) \forall x \in X$. However, when there exists at least a value $x \in X$ such that $\mu_{A}(x)<1-\nu_{A}(x)$, an extra parameter has to be taken into account: the hesitancy degree, $\tau_{A}(x)=1-\mu_{A}(x)-\nu_{A}(x)$, that represents the amount of lacking information in determining the membership of $x$ to $A$. If the hesitation degree is zero, the reciprocal relationship between membership and non-membership makes the latter one unnecessary in the formulation as it can be derived from the former.

In [11], Szmidt and Kacprzyk defined the intuitionistic fuzzy preference relation (IFPR) as a generalisation of the concept of the RPR.

Definition 6 (Intuitionistic Fuzzy PR (IFPR)): An intuitionistic fuzzy preference relation (IFPR) $B$ [12] on a finite set of alternatives $X$ is characterised by a membership function

$$
\mu_{B}: X \times X \rightarrow[0,1]
$$

and a non-membership function

$$
\nu_{B}: X \times X \rightarrow[0,1]
$$

such that

$$
0 \leq \mu_{B}\left(x_{i}, x_{j}\right)+\nu_{B}\left(x_{i}, x_{j}\right) \leq 1 \quad \forall\left(x_{i}, x_{j}\right) \in X \times X .
$$

An IFPR can be conveniently represented by a matrix $B=$ $\left(b_{i j}\right)$ with $b_{i j}=\left(\mu_{i j}, \nu_{i j}\right) \forall i, j=1,2, \ldots, n$. The value $\mu_{i j}=$ $\mu_{B}\left(x_{i}, x_{j}\right)$ can be interpreted as the certainty degree up to which $x_{i}$ is preferred to $x_{j}$, while the value $\nu_{i j}=\nu_{B}\left(x_{i}, x_{j}\right)$ represents the certainty degree up to which $x_{i}$ is non-preferred to $x_{j}$. When the following additional conditions are imposed:

- $\mu_{i i}=\nu_{i i}=0.5 \forall i$.

- $\mu_{j i}=\nu_{i j}, \nu_{j i}=\mu_{i j} \forall i, j$.

we refer to this IFPR as reciprocal and we will denote it as IRFPR. Notice that when the hesitancy degree function is the null function we have that $\mu_{i j}+\nu_{i j}=1 \quad \forall i, j$, and therefore the IRFPR $B=\left(b_{i j}\right)$ is mathematically equivalent to the APR $\left(\mu_{i j}\right)$, i.e. $B=\left(\mu_{i j}\right)$. Given an IFRPR, it is always possible to derive a RPR via the application of a score function [12], [13].

\section{Linguistic Preference Relation}

As aforementioned subjectivity, imprecision and vagueness in the articulation of opinions pervade real world decision applications [14], and individuals might feel more comfortable using words by means of linguistic labels or terms to articulate their preferences [15]. 
Let $\mathcal{L}=\left\{l_{0}, \ldots, l_{s}\right\}$ be a set of linguistic labels $(s \geq 2)$, with semantic underlying a ranking relation that can be precisely captured with a linear order, i.e., $l_{0}<l_{1}<\cdots<l_{s}$. Assuming that the number of labels is odd and the central label $l_{s / 2}$ stands for the indifference state when comparing two alternatives, the remaining labels are usually located symmetrically around that central assessment, which guarantees that a kind of reciprocity property holds as in the case of numerical preferences previously mentioned.

Definition 7 (Linguistic Preference Relation (LPR)): A LPR $P$ on a finite set of alternatives $X$ is characterised by a linguistic membership function $\mu_{P}: X \times X \longrightarrow \mathcal{L}, \mu_{P}\left(x_{i}, x_{j}\right)=$ $p_{i j} \in \mathcal{L}$.

Two main mathematical frameworks to represent LPRs in decision making have been proposed:

- LPR based on cardinal representation In this model each linguistic assessment is represented using a fuzzy number that is characterised by a membership function, with base variable the unit interval $[0,1]$, describing its semantic meaning. The membership function maps each value in $[0,1]$ to a degree of performance which represents its compatibility with the linguistic assessment [15].

- LPR based on ordinal representation In this case the semantics of the linguistic labels are established by assuming that in the set of linguistic terms $\mathcal{L}$ the labels are uniformly and symmetrically distributed around that central assessment $l_{s / 2}$, i.e., assuming the same discrimination levels on both sides of $l_{s / 2}$ and by considering that both terms $l_{i}$ and $l_{s-i}$ are equally informative.

Linguistic symbolic computational models have been defined to manage the ordinal linguistic information in the decision making problems [14]. The symbolic models work with the ordinal scales of the set of linguistic terms to combine linguistic information. There exit four different linguistic symbolic computational models based on ordinal scales, : (i) Linguistic symbolic computational model based on max-min operators [16], (ii) Linguistic symbolic model based on convex combination [17],(iii) Linguistic symbolic model based on virtual linguistic term set [18], (iv) Linguistic symbolic model based on the 2-tuple linguistic representation [19], which was introduced to avoid the loss of information that appears when the mentioned translation function in the linguistic symbolic model based on convex combination is applied.

This last model is built on the following linguistic 2-tuple representation definition:.

Definition 8: Let $\mathcal{L}$ be a linguistic term set and $\beta \in[0, s]$ a value supporting the result of a symbolic aggregation operation, then the 2-tuple that expresses the equivalent information to $\beta$ is obtained with the following function:

$$
\begin{array}{ll}
\Delta: & {[0, s] \longrightarrow S \times[-0.5,0.5)} \\
& \Delta(\beta)=\left(l_{i}, \alpha\right) \\
& i=\operatorname{round}(\beta) \\
& \alpha=\beta-i
\end{array}
$$

where "round" is the usual rounding operation, $l_{i}$ has the closest index label to " $\beta$ " and " $\alpha$ " is the value of the symbolic translation.

\section{Consistency}

As Cutello et al. stated in [20]: 'some individual opinions can be considered more consistent than other individual opinions' and they defined the explicit consistency as the'absence of explicit contradictions'. They also claimed that the problem of modelling rationality of individuals based only on their opinions over a finite and fixed set of alternatives expressed using FPRs can be considered a fuzzy concept. Indeed they modelled the fuzzy rationality measures, which are explicitly consistent, by establishing a collection of conditions that needed to be satisfied.

Consistency of RPRs has been modelled using the notion of transitivity in the pairwise comparison among any three alternatives. That is, if $x_{i}$ is preferred to $x_{j}\left(x_{i} \succ x_{j}\right)$ and this one to $x_{k}\left(x_{j} \succ x_{k}\right)$ then alternative $x_{i}$ should be preferred to $x_{k}\left(x_{i} \succ x_{k}\right)$, which is normally known as weak stochastic transitivity [21]. Furthermore any property that guarantees the transitivity of the preferences is called a consistency property [22].

A formal approach to model the multiplicative consistency for IVRPRs and IRFPRs has been proposed in [4] as a formal generalisation of the Tanino's multiplicative transitive property for RPRs [23] by applying Zadeh's Extension Principle [15] and the Representation Theorem of Fuzzy Sets [24].

The concept of consistent IVRPR is defined as follows:

Definition 9 (Multiplicative Consistent IVRPR): An IVRPR $B=\left(b_{i j}\right)=\left(\left[b_{i j}^{-}, b_{i j}^{+}\right]\right)$is consistent if and only if

$$
\forall i, j, k:\left\{\begin{array}{l}
b_{i j}^{-} b_{j k}^{-} b_{k i}^{-}=b_{i k}^{-} b_{k j}^{-} b_{j i}^{-} \\
b_{i j}^{+} b_{j k}^{+} b_{k i}^{+}=b_{i k}^{+} b_{k j}^{+} b_{j i}^{+}
\end{array}\right.
$$

In [4] the IRFPRs $R=\left(r_{i j}\right)=\left(\left\langle\mu_{i j}, \nu_{i j}\right\rangle\right)$ has been proved to be isomorphic to the IVRPRs $B=\left(b_{i j}\right)=\left(\left[\mu_{i j}, 1-\nu_{i j}\right]\right)$, therefore the multiplicative consistent IRFPR can be defined as follows:

Definition 10 (Multiplicative Consistent IRFPR): An IRFPR $R=\left(r_{i j}\right)=\left(\left\langle\mu_{i j}, \nu_{i j}\right\rangle\right)$ is consistent if and only if

$$
\forall i, j, k:\left\{\begin{array}{l}
\mu_{i j} \mu_{j k} \mu_{k i}=\mu_{i k} \mu_{k j} \mu_{j i} \\
\left(1-\nu_{i j}\right)\left(1-\nu_{j k}\right)\left(1-\nu_{k i}\right)= \\
\left(1-\nu_{i k}\right)\left(1-\nu_{k j}\right)\left(1-\nu_{j i}\right)
\end{array}\right.
$$

In the case of LPRs, the consistency property has been defined with different expressions depending on the linguistic approach used:

Definition 11 (Cardinal Additive Consistency of LPRs [25]): Given a LPR, $\tilde{P}=\tilde{p_{i j}}$ in which each linguistic preference degree has associated a triangular fuzzy membership function, i.e., $\tilde{p}_{i j}=\left(p_{i j}^{L}, p_{i j}^{M}, p_{i j}^{R}\right)$, then $\tilde{P}$ is additive consistent if and only if

$$
\forall i, j, k:\left\{\begin{array}{l}
p_{i j}^{L}+p_{j k}^{L}+p_{k i}^{R}=\frac{3}{2} \\
p_{i j}^{M}+p_{j k}^{M}+p_{k i}^{M}=\frac{3}{2} \\
p_{i j}^{R}+p_{j k}^{R}+p_{k i}^{L}=\frac{3}{2}
\end{array}\right.
$$


On the other hand, Tanino's multiplicative consistency for FPRs have been formally extended in [26] to the case of LPRs by means of Zadeh's Extension Principle [15] and the Representation theorem [24].

Definition 12 (MTP of a FLPR): A fuzzy linguistic preference relation $R=\left(r_{i j}\right)$ on a finite set of alternatives $X$ is multiplicative transitive if and only if

$$
\left.\begin{array}{rl}
\forall \alpha \in(0,1] \wedge & \forall i, k, j: \\
& r_{i j}^{\alpha-} \cdot r_{j k}^{\alpha-} \cdot r_{k i}^{\alpha-}=r_{i k}^{\alpha-} \cdot r_{k j}^{\alpha-} \cdot r_{j i}^{\alpha-} \\
& r_{i j}^{\alpha+} \cdot r_{j k}^{\alpha+} \cdot r_{k i}^{\alpha+}=r_{i k}^{\alpha+} \cdot r_{k j}^{\alpha+} \cdot r_{j i}^{\alpha+}
\end{array}\right\}
$$

Definition 13 (Ordinal Additive Consistency of LPRs [27]): Given a 2-tuple LPR $P=\left(p_{i j}\right)$ on a set of alternatives $X$, such that

$$
p_{i j}: X \times X \longrightarrow \mathcal{L} \times[-0.5,0.5)
$$

then $P$ will be considered consistent if for every three alternatives $x_{i}, x_{j}$ and $x_{k}$, the following condition holds

$$
p_{i k}=\Delta\left(\Delta^{-1}\left(p_{i j}\right)+\Delta^{-1}\left(p_{j k}\right)-\frac{s}{2}\right) \quad \forall i, j, k \in\{1, \ldots, n\} \text {. }
$$

\section{APPROACHES TO ESTIMATE MISSING INFORMATION UNDER UNCERTAINTY}

As aforementioned, in decision making there are circumstances when an expert is not always able to provide their preferences for a number of reasons ranging from lack of knowledge to lack of time. To model these situations the concept of incomplete PR was introduced in [28].

Definition 14: A function $f: X \longrightarrow Y$ is partial when not every element in the set $X$ necessarily maps to an element in the set $Y$. When every element from the set $X$ maps to one element of the set $Y$ then we have a total function.

Definition 15: A preference relation $P$ on a set of alternatives $\mathrm{X}$ with a partial membership function is an incomplete preference relation.

The approaches to tackle with incomplete information in GDM have been extensively studied in the last two decades [29]. Nevertheless the majority of them are aimed for the case of FPR [30]. Generally speaking, these proposals can be classified in two main categories depending on how they estimate the unknown information:

1) Iterative approaches: The missing information is estimated by means of indirect chains of known values. In each iteration the system recognizes the values that can be estimated at this time. The procedure stops when there are no more values to estimate.

2) Optimisation approaches: In this case a goal function, such as a consistency or consensus index, is optimised so as to estimate the missing values. In this sense, the two most relevant approaches for the case of FPRs are [31], [32]. These contributions have been used as the basis for other approaches developed for IFPRS, IVPRs and LPRs. On the other hand, other optimization methods are based on Saaty's assumption for Multiplicative PRs,
MPRs, regarding the exact functional relation between the preference values and the priority vector [33]. Therefore they directly calculate the ranking of the alternatives, also known as the weighting vector, instead of completing the PRs. However the missing PRs can be directly computed from this weighting vector.

In this section the main approaches developed in the literature to tackle incomplete IFRPRs, IVRPRs and LPRs are presented.

\section{A. Managing missing preference values in IVRPRs}

For the case of IVRPRs three methodologies have been reported: The first two use consistency properties to estimate the missing preference values [34], [35] whereas the last one [36] is based on the rough set theory [37].

- In [34], the so-called interval multiplicative transitivity property is introduced. This consistency property is built upon Tanino's [23] multiplicative consistency but applied for the optimistic and the pessimistic cases. Based on this consistency it estimates the missing preference values from the adjacent known ones.

- In [35] it is reported a framework applicable for various types of PRs, namely FPRs, MRPRs, 2-tuple LPRs and IVRPRs. To estimate the missing values of an IVRPRs the iterative, additive consistency based procedure proposed in [28] is applied independently to both the lower and upper bounds of the IVRPRs.

- In [36] it has been proposed a dominance-based rough set approach. The main novelty is that it considers three types of incomplete IVRPRs: (i) IVRPR with incomplete upper bound and complete lower bound, (ii) IVRPR with complete upper bound and incomplete lower bound, and (iii) IVRPR with both incomplete upper and lower bounds.

\section{B. Managing missing preference values in IFRPRs}

Three main iterative approaches have been considered for incomplete IFRPRs [12], [38], [4]. Furthermore an approach presented by $\mathrm{Xu}$ et al. in [39] to deal with missing interval value intuitionistic additive and multiplicative PRs (IVIFRPRs) is also analysed.

- In [12] the concept of multiplicative IFRPRs is defined based on the concept of multiplicative consistency of RPRs. The estimation of the missing values is accomplished by means of an iterative method driven by the multiplicative consistency.

- The work proposed in [38] presents a completion method also based on the multiplicative consistency property. This method uses an indirect chain of known values to estimate the unknown ones, applying the multiplicative consistency independently to the $\mu_{i j}$ and $\nu_{i j}$.

- In [4] it has been proposed a GDM process with consensus in which the missing values of the IFPPRs are estimated following an iterative procedure built upon the one in [28]. This method is based on the multiplicative consistency property presented before in Definition 10 . 
- Xu et al. introduce in [39] the additive and the multiplicative consistent incomplete IVIFRPRs and define the concept of acceptable incomplete interval-valued intuitionistic fuzzy preference relation. In this contribution they also propose two procedures for completing the acceptable incomplete interval-valued intuitionistic based on the arithmetic average and the geometric mean, respectively.

- In [40] an approach to multiattribute decision making with incomplete attribute weight information where individual assessments are provided as IVIFRPRs has been proposed. By means of optimization models, this methodology derives a linear program for determining attribute weights.

\section{Managing missing preference values in LPRs}

In this subsection we present the methodologies classified depending on the linguistic frameworks they use: (i) 2-tuple LPRs [35], [41], [42]; (ii) LPRs based on virtual linguistic term sets [43], [44]; and (iii) LPRs based on a cardinal approach [45], [25].

(i) 2-tuple LPRs.

a) Alonso et al. in [35] propose a method which converts the 2- tuple LPR into an RPR applying the transformation functions between 2-tuples and RPRs proposed in [19]. Then it estimates the missing values using the additive transitivity property for RPRs. Once the RPRs is completed, it is transformed back to the corresponding 2-tuple LPR.

b) Also Alonso et al. in [27] apply the linguistic additive consistency property to estimate the missing 2-tuple linguistic values and design an iterative procedure built upon the one proposed in [28] for RPRs. This approach was later used in [41] to define an additive consistency measure of the information provided by each expert to assign importance degrees to experts in the aggregation process. Furthermore in [42] it has been presented an application of this methodology to estimate unknown users' preferences in fuzzy linguistic recommender systems.

(ii) LPRs based on Virtual linguistic term sets.

a) $\mathrm{Xu}$ in [43] proposes an additive transitivity property based method to estimate missing LPRs assessed on virtual linguistic term sets. This author also propose in [46] and in [47] completion methods based on the multiplicative transitivity.

b) Hsu et al. in [44] present an alternative additive transitivity property based estimation method of missing LPRs assessed on virtual linguistic term sets for which they propose three ways of pairwise comparisons: horizontal, vertical and oblique.

(iii) LPRs based on a cardinal approach.

a) $\mathrm{Li}$ et al. [45] propose an extension of the well known LINMAP method [48] to deal with decision making problems with fuzzy linguistic information. Each al- ternative is assessed on the basis of its distance to a fuzzy positive ideal solution (FPIS) which is unknown, using a new method to calculate the distance between trapezoidal fuzzy number scores. The FPIS and the weights of attributes are then estimated using a linear programming model guided by the consistency and inconsistency criteria. The distance of each alternative to the FPIS is calculated to determine the ranking order of all alternatives.

b) Wang et al. [25] present an approach which uses triangular membership function to model linguistic information and that is driven by the additive consistency property.

\section{CONCLUSIONS AND FUTURE WORK}

Hesitance and uncertainty is inherently related to human beings when making decisions. In formal group decision making approaches this hesitance needs to be addressed allowing the experts to provide their preferences without forcing them to give an exact value. To that aim different families of PRs have been proposed namely: IFRPRs, IVRPRs and LPRs. However, in real situations, the experts might not be able to differentiate the degree up to which one alternative is preferred to another providing PRs with missing values. In this contribution we have analysed the research efforts carried out in order to estimate missing information when the experts are allowed to enunciate their preferences by means of the PRs mentioned above. Those method are mainly guided by consistency criteria, formally extended for RPRs following Zadeh's Extension Principle and the Representation Theorem. These approaches can be widely classified in two main groups: 1) Iterative approaches 2) Optimisation approaches. Apart from the PRs that have been subject of study in this contribution there are other promising preference representation frameworks: Hesitant fuzzy PRs [49] (HFPRs) based on Torra's Hesitant Fuzzy sets [50] and LPRs modelled by Interval Type-2 fuzzy sets [51] (IT2PRs). Indeed, this second framework deserves especial attention since it allows to model the uncertainty not only in the preference degree but also in the meaning of the linguistic label, since, as it has been stated by Mendel in [5], same words mean different things for different people. Nevertheless, in spite of being very useful to reflect uncertainty in the experts' opinions, as far as we are concerned, there are no formal approaches that deal with missing information for these types of IT2PRs. Therefore as future work, we plan to develop approaches based on consistency to deal with missing information for HPRs, and IT2PRs.

\section{REFERENCES}

[1] I. Millet, "The effectiveness of alternative preference elicitation methods in the analytic hierarchy process," Journal of Multi-Criteria Decision Analysis, vol. 6, no. 1, pp. 41-51, 1997.

[2] D. H. Ebenbach and C. Moore, "Incomplete information, inferences, and individual differences: The case of environmental judgments," Organizational Behavior and Human Decision Processes, vol. 81, no. 1, pp. $1-27,2000$.

[3] F. J. Carmone, A. Kara, and S. H. Zanakis, "A monte carlo investigation of incomplete pairwise comparison matrices in AHP.," European Journal of Operational Research, vol. 102, no. 3, pp. 538-553, 1997. 
[4] J. Wu and F. Chiclana, "Multiplicative consistency of intuitionistic reciprocal preference relations and its application to missing values estimation and consensus building," Knowledge-Based Systems, vol. 71, no. 0 , pp. 187 - 200, 2014.

[5] J. Mendel and D. Wu, Perceptual Computing: Aiding People in Making Subjective Judgments. Wiley-IEEE Press, 2010.

[6] P. Fishburn, Utility theory for decision making. Melbourne, FL: Krieger, 1979.

[7] J. Bezdek, B. Spillman, and R. Spillman, "A fuzzy relation space for group decision theory," Fuzzy Sets and Systems, vol. 1, no. 4, pp. 255268, 1978

[8] J. M. Mendel, Uncertain rule-based fuzzy logic systems: introduction and new directions. Prentice-Hall PTR, 2001.

[9] G. J. Klir and T. A. Folger, Fuzzy sets, uncertainty, and information. Prentice-Hall International., 1992.

[10] K. T. Atanassov, "Intuitionistic fuzzy sets," Fuzzy Sets and Systems, vol. 20, no. 1, pp. 87-96, 1986.

[11] E. Szmidt and J. Kacprzyk, "Using intuitionistic fuzzy sets in group decision making," Control and Cybernetics, vol. 31, no. 4, pp. 1037$1053,2002$.

[12] $\mathrm{Z} . \mathrm{Xu}$, "Intuitionistic preference relations and their application in group decision making," Information Sciences, vol. 177, no. 11, pp. $2363-$ 2379, 2007

[13] J. Wu and F. Chiclana, "Non-dominance and attitudinal prioritisation methods for intuitionistic and interval-valued intuitionistic fuzzy preference relations," Expert Systems with Applications, vol. 39, no. 18, pp. 13409-13416, 2012.

[14] F. Herrera, S. Alonso, F. Chiclana, and E. Herrera-Viedma, "Computing with words in decision making: foundations, trends and prospects," Fuzzy Optimization and Decision Making, vol. 8, no. 4, pp. 337-364, 2009.

[15] L. A. Zadeh, "The concept of a linguistic variable and its application to approximate reasoning- i," Information Sciences, vol. 8, pp. 199-249, 1975.

[16] R. Yager, "A new methodology for ordinal multiobjective decisions based on fuzzy sets," Decision Sciences, vol. 12, pp. 589-600, 1981.

[17] F. Herrera, E. Herrera-Viedma, and J. L. Verdegay, "Direct approach processes in group decision making using linguistic owa operators.," Fuzzy Sets and Systems, no. 79, pp. 175-190, 1996.

[18] Z. Xu, "A method based on linguistic aggregation operators for group decision making with linguistic preference relations," Information Sciences, vol. 166, no. 1-4, pp. 19-30, 2004.

[19] F. Herrera and L. Martinez, "A 2-tuple fuzzy linguistic representation model for computing with words," IEEE Transactions on Fuzzy Systems, vol. 8, pp. 746-752, 2000.

[20] V. Cutello and J. Montero, "Fuzzy rationality measures," Fuzzy Sets and Systems, vol. 62, no. 1, pp. 39 - 54, 1994.

[21] R. D. Luce and P. Suppes, Preferences, utility and subject probability. Handbook of mathematical psychology, vol. 3. 1965.

[22] F. Chiclana, E. Herrera-Viedma, S. Alonso, and F. Herrera, "Cardinal consistency of reciprocal preference relations: A characterization of multiplicative transitivity," IEEE Transactions on Fuzzy Systems, vol. 17, no. 1, pp. 14-23, 2009.

[23] T. Tanino, "Fuzzy preference orderings in group decision making," Fuzzy Sets and Systems, vol. 12, pp. 117-131, 1984.

[24] L. A. Zadeh, "Fuzzy sets," Information and Control, vol. 8, no. 3, pp. 338-357, 1965.

[25] T.-C. Wang and Y.-H. Chen, "Incomplete fuzzy linguistic preference relations under uncertain environments," Information Fusion, vol. 11, no. 2, pp. 201-207, 2010.

[26] F. Chiclana, J. Wu, and E. Herrera-Viedma, "Consistency based estimation of fuzzy linguistic preferences. the case of reciprocal intuitionistic fuzzy preference relations," in Fuzzy Systems (FUZZ-IEEE), 2014 IEEE International Conference on, pp. 273-278, July 2014.

[27] S. Alonso, F. Cabrerizo, F. Chiclana, F. Herrera, and E. Herrera-Viedma "Group decision making with incomplete fuzzy linguistic preference relations," Int. J. Intell. Syst, vol. 24, no. 2, pp. 201-222, 2009.

[28] E. Herrera-Viedma, F. Chiclana, F.Herrera, and S. Alonso, "Group decision-making model with incomplete fuzzy preference relations based on additive consistency," IEEE Transactions on Systems, Man, and Cybernetics, Part B: Cybernetics, vol. 37, no. 1, pp. 176-189, 2007.

[29] R. Urena, F. Chiclana, J. Morente-Molinera, and E. Herrera-Viedma, "Managing incomplete preference relations in decision making: A review and future trends," Information Sciences, vol. 302, no. 0, pp. 14 - 32, 2015.

[30] R. Urena, F. Chiclana, S. Alonso, J. A. Morente-Molinera, and E. Herrera-Viedma, "On incomplete fuzzy and multiplicative preference relations in multi-person decision making," Procedia Computer Science, vol. 31, no. 0, pp. 793 - 801, 2014. 2nd International Conference on Information Technology and Quantitative Management, \{ITQM 2014

[31] G. Zhang, Y. Dong, and Y. Xu, "Linear optimization modeling of consistency issues in group decision making based on fuzzy preference relations," Expert Systems with Applications, vol. 39, pp. 2415-2420, 2012.

[32] M. Fedrizzi and S. Giove, "Incomplete pairwise comparison and consistency optimization," European Journal of Operational Research, vol. 183, no. 1, pp. 303-313, 2007.

[33] T. L. Saaty, The analytic hierarchy process. McGraw-Hill, 1980.

[34] S. Genc, F. E. Boran, D. Akay, and Z. Xu, "Interval multiplicative transitivity for consistency, missing values and priority weights of interval fuzzy preference relations.," Information Sciences, vol. 180, no. 24 , pp. 4877 - 4891., 2010.

[35] S. Alonso, F. Chiclana, F. Herrera, E. Herrera-Viedma, J. Alcalá-Fdez, and C. Porcel, "A consistency-based procedure to estimate missing pairwise preference values," International Journal of Intelligent Systems, vol. 23, no. 2, pp. 155-175, 2008.

[36] X. Yang, D. Yu, J. Yang, and L. Wei, "Dominance-based rough set approach to incomplete interval-valued information system," Data \& Knowledge Engineering, vol. 68, no. 11, pp. 1331-1347, 2009.

[37] Z. Pawlak, Rough sets: theoretical aspects of reasoning about data. Kluwer Academic Publishers, 1992.

[38] Z. Xu, X. Cai, and E. Szmidt, "Algorithms for estimating missing elements of incomplete intuitionistic preference relations," International Journal of Intelligent Systems, vol. 26, no. 9, pp. 787-813, 2011.

[39] Z. Xu and X. Cai, "Incomplete interval-valued intuitionistic fuzzy preference relations," International Journal of General Systems, vol. 38, no. 8, pp. 871-886, 2009

[40] Z. Wang, K. W. Li, and W. Wang, "An approach to multiattribute decision making with interval-valued intuitionistic fuzzy assessments and incomplete weights," Information Sciences, vol. 179, no. 17, pp. 3026 $-3040,2009$.

[41] F. J. Cabrerizo, R. Heradio, I. J. Pérez, and E. Herrera-Viedma, "A selection process based on additive consistency to deal with incomplete fuzzy linguistic information," Journal of Universal Computer Science, vol. 16, no. 1, pp. 62-81, 2010.

[42] C. Porcel and E. Herrera-Viedma, "Dealing with incomplete information in a fuzzy linguistic recommender system to disseminate information in university digital libraries," Knowledge-Based Systems, vol. 23, pp. 3239, 2010.

[43] Z. S. Xu, "Incomplete linguistic preference relations and their fusion," Information Fusion, vol. 7, no. 3, pp. 331-337, 2006.

[44] S.-C. Hsu and T.-C. Wang, "Solving multi-criteria decision making with incomplete linguistic preference relations," Expert Systems with Applications, vol. 38, no. 9, pp. 10882-10888, 2011.

[45] D.-F. Li and T. Sun, "Fuzzy linmap method for multiattribute group decision making with linguistic variables and incomplete information," International Journal of Uncertainty, Fuzziness and Knowledge-Based Systems, vol. 15, no. 2, pp. 153-173, 2007.

[46] Z. S. Xu, "Group decision and negotiation," A Practical Procedure for Group Decision Making under Incomplete Multiplicative Linguistic Preference Relations, vol. 15, no. 6, pp. 581-590, 2006.

[47] M. Xia, Z. Xu, and Z. Wang, "Multiplicative consistency-based decision support system for incomplete linguistic preference relations," International Journal of Systems Science, vol. 45, no. 3, pp. 625-636, 2014

[48] V. Srinivasan and A. Shocker, "Linear programming techniques for multidimensional analysis of preference," Psychometrika, vol. 38, pp. 337342,1973

[49] H. Liao, Z. Xu, and M. Xia, "Multiplicative consistency of hesitant fuzzy preference relation and its application in group decision making," International Journal of Information Technology and Decision Making, vol. 13, no. 01, pp. 47-76, 2014

[50] V. Torra, "Hesitant fuzzy sets," International Journal of Intelligent Systems, vol. 25, pp. 529-539, 2010.

[51] J. Mendel, R. John, and F. Liu, "Interval type-2 fuzzy logic systems made simple," Fuzzy Systems, IEEE Transactions on, vol. 14, pp. 808821, Dec 2006 